\title{
BMJ Open Sodium selenate as a disease-modifying treatment for progressive supranuclear palsy: protocol for a phase 2, randomised, double-blind, placebo- controlled trial
}

\author{
Lucy Vivash (D) , ${ }^{1,2,3,4}$ Kelly L Bertram, ${ }^{1,2}$ Charles B Malpas, ${ }^{1,2,5}$ \\ Cassandra Marotta, ${ }^{1,2}$ lan H Harding, ${ }^{1}$ Scott Kolbe, ${ }^{1}$ Joanne Fielding, ${ }^{1}$ \\ Meaghan Clough, ${ }^{1}$ Simon J G Lewis, ${ }^{6}$ Stephen Tisch, ${ }^{7}$ Andrew H Evans, ${ }^{3}$ \\ John D O'Sullivan, ${ }^{8,9}$ Thomas Kimber, ${ }^{10}$ David Darby, ${ }^{1,2,11,12}$ Leonid Churilov, ${ }^{4}$ \\ Meng Law, ${ }^{1,13}$ Christopher M Hovens, ${ }^{14}$ Dennis Velakoulis, ${ }^{15,16}$ \\ Terence J O'Brien ${ }^{1,2,3,4}$
}

To cite: Vivash L, Bertram KL, Malpas CB, et al. Sodium selenate as a disease-modifying treatment for progressive supranuclear palsy: protocol for a phase 2, randomised, double-blind, placebocontrolled trial. BMJ Open 2021;11:e055019. doi:10.1136/ bmjopen-2021-055019

- Prepublication history and additional supplemental material for this paper are available online. To view these files, please visit the journal online (http://dx.doi.org/10.1136/ bmjopen-2021-055019).

Received 01 July 2021 Accepted 14 October 2021

Check for updates

(C) Author(s) (or their employer(s)) 2021. Re-use permitted under CC BY-NC. No commercial re-use. See rights and permissions. Published by BMJ.

For numbered affiliations see end of article.

Correspondence to

Dr Lucy Vivash;

lucy.vivash@monash.edu

\section{ABSTRACT}

Introduction Progressive supranuclear palsy (PSP) is a neurodegenerative disorder for which there are currently no disease-modifying therapies. The neuropathology of PSP is associated with the accumulation of hyperphosphorylated tau in the brain. We have previously shown that protein phosphatase 2 activity in the brain is upregulated by sodium selenate, which enhances dephosphorylation. Therefore, the objective of this study is to evaluate the efficacy and safety of sodium selenate as a disease-modifying therapy for PSP.

Methods and analysis This will be a multi-site, phase $2 \mathrm{~b}$, double-blind, placebo-controlled trial of sodium selenate. 70 patients will be recruited at six Australian academic hospitals and research institutes. Following the confirmation of eligibility at screening, participants will be randomised (1:1) to receive 52 weeks of active treatment (sodium selenate; $15 \mathrm{mg}$ three times a day) or matching placebo. Regular safety and efficacy visits will be completed throughout the study period. The primary study outcome is change in an MRI volume composite (frontal lobe+midbrain-3rd ventricle) over the treatment period. Analysis will be with a general linear model (GLM) with the MRI composite at 52 weeks as the dependent variable, treatment group as an independent variable and baseline MRI composite as a covariate. Secondary outcomes are change in PSP rating scale, clinical global impression of change (clinician) and change in midbrain mean diffusivity. These outcomes will also be analysed with a GLM as above, with the corresponding baseline measure entered as a covariate. Secondary safety and tolerability outcomes are frequency of serious adverse events, frequency of down-titration occurrences and frequency of study discontinuation. Additional, as yet unplanned, exploratory outcomes will include analyses of other imaging, cognitive and biospecimen measures.

Ethics and dissemination The study was approved by the Alfred Health Ethics Committee (594/20). Each participant or their legally authorised representative and
Strengths and limitations of this study

- A large placebo-controlled, double-blind randomised controlled trial of a new drug treatment for progressive supranuclear palsy.

- The collection of a large body of clinical, cognitive and imaging data will result in a highly characterised prospective patient cohort, which will inform the field for future selection of clinical trial outcome measures.

- The use of both established and novel diagnostic methods may result in the validation of new diagnostic and prognostic approaches for future application in both clinical and research settings.

- The long treatment duration could impact participant completion due to disease progression.

their study partner will provide written informed consent at trial commencement. The results of the study will be presented at national and international conferences and published in peer-reviewed journals.

Trial registration number Australian New Zealand Clinical Trials Registry (ACTRN12620001254987).

\section{INTRODUCTION}

Progressive supranuclear palsy (PSP) is a rare, rapidly progressing, neurodegenerative movement disorder. Richardson's syndrome (PSP-RS) is the classical and most common form of PSP. It is a Parkinsonian disorder, characterised by vertical oculomotor (OM) dysfunction, frontal dysexecutive dysfunction and postural instability and falls. The prevalence rate is approximately 6 per 100000 people, ${ }^{1}$ with typical survival being $7-8$ years from symptoms onset. ${ }^{2}$ There are currently 
no approved disease-modifying treatments for PSP, and none of the limited number of international clinical trials that have been conducted to date have been successful. ${ }^{34}$ Therefore there is a major unmet clinical need for the treatment of PSP.

PSP is considered pathologically in the group of diseases termed 'tauopathies' which are characterised by the accumulation of hyperphosphorylated inclusions of the microtubule-associated protein tau, which in patients with PSP initially accumulates in the basal ganglia and deep nuclei of the cerebellum, before spreading to other cortical and subcortical brain regions. ${ }^{5}$ Thus, hyperphosphorylated tau is a potential target for the treatment of PSP that warrants exploration in randomised clinical trials.

Pharmacological reduction of tau hyperphosphorylation may be achieved by two broad approaches: (1) inhibition of tau phosphorylation through action on serine/ threonine kinases, the group of enzymes responsible for phosphorylation, or (2) increasing dephosphorylation of hyperphosphorylated tau by activating tau serine/threonine phosphatases, the group of enzymes that dephosphorylate proteins. Protein phosphatase 2 (PP2A) is the major tau phosphatase in the brain accounting for more than $70 \%$ of brain phosphatase activity, and thus stimulation of its activity presents a compelling strategy for reducing hyperphosphorylated tau. ${ }^{6}$ PP2A is colocalised with tau, and in many neurodegenerative diseases, reduced PP2A activity is observed alongside reductions in tau dephosphorylation. ${ }^{7-9}$

The trace metal selenium is an essential element in humans. It is present in low concentrations in the environment and in foods such as Brazil nuts. Previously, dietary supplementation with selenium has been reported to have potential chemopreventive benefits, ${ }^{10}$ however, this has been limited to selenium, with the potential therapeutic benefits of other selenium compounds yet to be comprehensively investigated. Work by our team and others is amassing a large growing body of preclinical, and emerging clinical data, demonstrating that sodium selenate, a selenium salt, may have potential as a therapeutic agent. Our work has demonstrated that through the activation of PP2A increasing rates of dephosphorylation, sodium selenate has potential as a disease-modifying treatment in neurodegenerative diseases associated with hyperphosphorylated tau, as well as epilepsy and traumatic brain injury. ${ }^{11-21}$ We have reported sodium selenate provides benefits in a range of animal models of disease including Alzheimer's disease (AD) ${ }^{13}{ }^{22}$ cancer, ${ }^{12}$ traumatic brain injury ${ }^{111819}$ and epilepsy. ${ }^{15161821}$ Moreover, these benefits were specific to sodium selenate, with no benefits observed with other selenium species, and were only observed when supranutritional doses were administered. ${ }^{1723}$

Three clinical trials completed by our team have demonstrated the safety and tolerability of chronic dosing with sodium selenate in clinical populations other than PSP. The first study was a phase 1 safety and tolerability study in patients with castration-resistant prostate cancer. Safety and tolerability was good, with doses up to $60 \mathrm{mg}$ per day being well-tolerated, and dose-limiting toxicity observed at $90 \mathrm{mg}$ per day. ${ }^{12}$ A phase 2a trial in patients with mild-moderate $\mathrm{AD}(\mathrm{n}=40)$, confirmed the safety and tolerability of sodium selenate $(30 \mathrm{mg} /$ day $)$ at this dose over 6 months. ${ }^{1723}$ In exploratory efficacy analyses, diffusion tensor imaging (DTI) measures indicated relatively less neurodegeneration in the treatment group compared with the combined placebo/nutritional dose group. Widespread neurodegeneration was observed on DTI, with the corpus callosum showing the most severe degeneration. ${ }^{17}$ Increased selenium levels in serum and cerebrospinal fluid (CSF) of the treatment group demonstrated that the sodium selenate was able to cross the blood-brain barrier and enter the central nervous system. ${ }^{23}$ The degree of cognitive decline (measured on the Mini-Mental State Examination) over the 24 weeks of treatment inversely correlated with selenium levels in the CSF, suggesting neuroprotective efficacy may be dependent on greater drug exposure levels. ${ }^{23}$ Long-term safety and tolerability were demonstrated in an open-label extension study, where patients with $\mathrm{AD}$ received sodium selenate $\left(30 \mathrm{mg} /\right.$ day) for up to 23 months. ${ }^{24}$ Furthermore, cognitive decline measured on the Alzheimer's Disease Assessment Scale Cognitive Subscale and other psychometric scales showed substantially less decline than would be predicted based on the natural progression of $\mathrm{AD}^{24}$

Most recently we completed a small phase $1 \mathrm{~b}$ open-label study of sodium selenate treatment at doses up to $45 \mathrm{mg}$ per day in patients with possible behavioural variant frontotemporal dementia (bvFTD), another neurodegenerative disease characterised by hyperphosphorylated tau (ACTRN12617001218381). Safety and tolerability were again excellent, with all patients $(n=12)$ completing the study. Adverse events were mild and similar to those reported in previous studies, the most common being nail changes (58\%) and hair loss (42\%). Exploratory efficacy measures (MRI, cognition, behaviour) suggested slowing of disease progression in a subgroup of participants $(\mathrm{n}=7)$, with the other subgroup $(\mathrm{n}=4)$ showing substantial disease progression. This division of 'responders' and 'non-responders' is in keeping with the known incidence of tau and non-tau pathology in bvFTD. ${ }^{25}$ Informed by these results we have recently commenced recruitment of a phase $2 \mathrm{~b}$ placebo-controlled randomised controlled trial of sodium selenate as a treatment for bvFTD (ACTRN12620000236998 ${ }^{26}$ ).

These prior experiences in neurodegenerative diseases defined by aggregation of hyperphosphorylated tau have informed this current study, a multi-centred placebocontrolled, double-blind randomised controlled trial of sodium selenate as a treatment for PSP. The present study closely mirrors our phase $2 \mathrm{~b}$ trial in bvFTD in overall trial design, schedule of assessments (including safety assessments and outcomes, cognitive measures and exploratory biomarkers) and numerous exclusion criteria. ${ }^{26}$ 


\section{METHODS AND ANALYSIS}

This is a multi-site, phase 2, double-blind, randomised, placebo-controlled trial to assess the safety and efficacy of sodium selenate as a treatment for PSP (RS). Participants will receive either sodium selenate $(15 \mathrm{mg}$, three times a day) or placebo for 52 weeks. Seventy patients will be recruited in to this study. The study will be conducted at six centres in Melbourne, Sydney, Brisbane and Adelaide. The study is funded by the Australian Medical Research Future Fund (GNT1200254). Ethics approval was granted by Alfred Health Human Research Ethics Committee, Melbourne (594/20). The trial is registered with the ANZCTR (ACTRN12620001254987). The study commenced recruitment in July 2021 and is anticipated to complete (last patient last visit) in March 2025.

\section{Outcomes}

The primary outcome measure will be a change in MRI composite volume (frontal lobe+midbrain-3rd ventricle) over the 52-week treatment period.

The secondary outcome measures (safety) will be the rate and severity of adverse events and the rate of study withdrawal. Secondary efficacy outcome variables will be the change in PSP symptoms as measured by the PSP rating scale total score, change in disease severity as measured by the clinical global impression of change (CGI-C) total score and change in mean diffusivity (MD) in the midbrain calculated from diffusion-weighted MRI over the 52 weeks of treatment.

Numerous exploratory outcomes will also be measured including changes in protein biomarkers (total-tau, phospho-tau and neurofilament light chain (NfL)) in CSF, plasma and serum, changes in cognitive measures, changes in OM functioning (including measures of motor and cognitive functioning), changes in other structural and functional neuroimaging metrics including regional volumes and cortical thickness, advanced MRI (diffusion imaging, quantitative susceptibility mapping, resting state functional MRI) and tau-binding positron emission tomography (PET) and pharmacokinetic modelling. Advanced statistical modelling will be investigated to identify baseline predictors of treatment response and non-response. Finally, correlation analyses will be used to investigate the relationships between objective biomarkers and the presence and progression of symptoms.

\section{Eligibility criteria}

\section{Inclusion criteria}

Participants will be aged over 40, have a diagnosis of probable PSP-RS ${ }^{27}$ and symptoms present for $<5$ years at the time of screening. The participant must live in the community and have at least 10 contact hours per week with a responsible carer. The carer should be capable of ensuring the participant's compliance with the medication and study, and complete questionnaires about the participant's symptoms throughout the study. Participants must be using effective contraception for the duration of the trial. Participants must have a lumbar puncture (LP) and MRI performed during screening. The structural brain MRI must be not inconsistent with a diagnosis of PSP-RS with no other gross structural abnormalities indicating another neurological disorder. Written informed consent must be obtained from the participant or their legally authorised representative (as required by local laws and regulations), and the participant's carer.

\section{Exclusion criteria}

Participants will be excluded based on: history of substance use disorder (including alcohol and cannabis); previous participation in an interventional clinical trial (within 3 months of screening), with the exception of prior exposure to sodium selenate; known sensitivity to selenium, sodium selenate, any medicine or vitamin containing sodium selenate, similar agents or any of the excipients (including microcrystalline cellulose) used; likely non-compliance with the trial visit schedule or trial medication; evidence or history of neurological, psychiatric or other illness that could contribute to PSP-like symptoms; known history of familial $\mathrm{AD}$ or genetic variant that confers likelihood of another neurodegenerative condition (eg, PRNP, SYNJ1, PSEN1, C9ORF72 expansion); significant comorbid medical (including unstable diabetes) or neurological disease, with the exception of PSP, that is not adequately controlled by therapy and may interfere with the patient's ability to complete the study or affect the patient's cognitive performance; contraindication to MRI or LP; significant impairment of renal, hepatic or haematological function; participant is or has (within 6 weeks of the screening visit) taken any of the following: N-methyl-D-aspartate (NMDA) receptor antagonists, oral and/or injectable steroids, digoxin, phenobarbitone or warfarin; commencement or titration of other medications known to have an effect on mood or cognition within the 4 weeks prior to screening, including anticholinergics, hypnotics, sedatives, anxiolytics, antidepressants, antiepileptics, antipsychotics, memoryenhancing drugs, nutraceuticals and other supplements which contain selenium and dopaminergic drugs. ${ }^{26}$

\section{Intervention, randomisation and blinding}

Once consent has been obtained, each participant will be provided a unique screening number. On completion of all screening assessments and confirmation of eligibility, a sequential randomisation number will be generated from within the redcap electronic Case Report Form ((eCRF) subject to entry of key data into the eCRF). Once the randomisation number has been provided to the unblinded site pharmacist, the unblinded pharmacist will dispense the drug/placebo in accordance with the randomisation schedule. Participants will be allocated at a ratio of 1:1 either sodium selenate or placebo for 52 weeks. Each tablet will contain $5 \mathrm{mg}$ of drug or placebo. Participants will titrate from an initial dose of two tablets $(10 \mathrm{mg})$ three times a day, increasing to three tablets $(15 \mathrm{mg})$ three times a day at week 4 , subject to tolerability. If an adverse event occurs that is potentially related to the 
study drug administration, treatment may be temporarily interrupted, at the discretion of the investigator, a single within-subject dose reduction, to $10 \mathrm{mg}$ three times a day will also be allowed. A further down-titration to $5 \mathrm{mg}$ three times a day will require consultation with and approval by the medical monitor.

Throughout the course of the study, the participant, their study partner and all site staff (with the exception of the pharmacy team) will remain blinded to treatment allocation. Emergency unblinding of individual participants may performed by site pharmacy staff or by accessing individual unblinding envelopes kept on site. The data safety monitoring board (DSMB) will remain blinded to treatment allocation when reviewing safety data. Unblinding of data may be requested by the DSMB in the event of unexpected adverse events for which unblinding is deemed necessary for the assessment of potential causality. ${ }^{26}$

\section{Procedures and assessments}

Table 1 outlines the testing and procedures that participants will undergo which is based on our other phase 2 trial in bvFTD. ${ }^{26}$ Briefly, at screening, the participant will be reviewed to ensure they meet all the inclusion criteria and none of the exclusion criteria. Neuroimaging (MRI) will follow to corroborate the PSP-RS diagnosis. At baseline, the participant's eligibility will be confirmed by repeated review of the inclusion and exclusion criteria. Baseline tau-PET, CSF sampling and cognitive and symptomatic assessment will be completed. OM testing will be performed in participants who consent to the OM substudy. Finally, the participant will receive their first dose of the study drug $(10 \mathrm{mg})$ in the clinic and multiple blood draws taken for pharmacokinetic analysis.

Safety phone calls to monitor for adverse events will be completed. Subject to tolerability, participants will be uptitrated to three tablets $(15 \mathrm{mg}$ three times a day) at week 4 . Both solicited and unsolicited adverse events that occur between clinic visits will be recorded in diary cards given to participants.

As detailed in table 1, regular study visits will occur to assess participant safety and well-being, and to ensure treatment compliance and continued supply of the study medication. Additionally at weeks 26 and 52, the cognitive and symptomatic assessment (as well as OM testing for those in the substudy) will be repeated. Repeated neuroimaging (MRI and tau-PET) and CSF sampling for biomarker analyses will occur in the 2 weeks prior to the week 52 clinical visit. A final safety visit will be completed 4 weeks after the end of treatment (week 52) visit.

\section{Measures}

Neuroimaging

MRIs will be acquired during the screening period and week 52. The following sequences are included in the MRI protocol: whole-brain volumetric 3D T1-weighted $(0.8 \mathrm{~mm}$ isotropic voxels), T2-weighted $(0.8 \mathrm{~mm}$ isotropic) and T2-weighted fluid-attenuated inversion recovery (FLAIR; $0.8 \mathrm{~mm}$ isotropic) images; multi-echo T2*-weighted images $(0.8 \mathrm{~mm}$ isotropic) for susceptibility mapping; multi-shell diffusion-weighted imaging (DWI; $2 \mathrm{~mm}$ isotropic) and multi-band resting state functional MRI (2.4 mm isotropic).

The primary study outcome will be the change in MRI composite (frontal lobe+midbrain-3rd ventricle) volume, measured using T1-weighted structural MRI, from baseline to 52 weeks. Change in composite volume will be measured using the method described by Höglinger et $a l .^{28}$ Volumes will be corrected for intracranial volume (ICV), and normalised to the mean ICV for the whole study population.

Tau PET using the second-generation specific taubinding radiotracer $\left[{ }^{18} \mathrm{~F}\right]$-PI2620 (Life Molecular Imaging, Berlin, Germany) will be performed at baseline and in the 2 weeks prior to week 52. A dynamic 3D acquisition $(10 \times 30 \mathrm{~s}, 5 \times 60 \mathrm{~s}, 10 \times 300 \mathrm{~s})$ will begin on intravenous injection of $185 \mathrm{MBq}( \pm 10 \%)$ of the tracer.

\section{Cognitive and symptomatic battery}

The cognitive and symptomatic battery, consisting of the following scales will be administered at three timepoints throughout the study, baseline, week 26 and week 52.

\section{PSP rating scale}

The PSP rating scale is a clinician-administered quantitative measure of disability in participants with PSP. ${ }^{29}$ The PSP rating scale comprises 28 items in 6 areas. The available total score ranges from 0 (normal) to 100 . The six areas assessed are: daily activities, mentation, bulbar, ocular motor, limb and gait.

\section{Clinical global impression of change}

The CGI-C scale is a clinician-administered scale which measures the change in the patient's clinical status from a specific point in time. ${ }^{30}$ Using a 7 -point scale, ranging from 1 (very much improved) to 7 (very much worse), with a score of 4 indicating no change.

\section{Trail Making Test $A$ and $B$}

The Trail Making Test is a test of visual attention, processing speed and task switching. There are two parts to the test, $\mathrm{A}$ and $\mathrm{B}$, where the participant is asked to draw lines to connect 25 targets as quickly as possible without making any mistakes. In the first part of the test, the participant is asked to connect sequential numbers in order (1-25). In the second part the targets alternate between numbers and letters (1, A, 2, B, etc). Part A is a measure of processing speed, while part B measures executive function. The number of seconds taken to complete each path are the scores for this assessment. ${ }^{31}$

\section{Frontal assessment battery}

The frontal assessment battery is a battery of six short tests which examines executive function. ${ }^{32}$ The tests consist of (1) similarities, whereby the participant must identify in what way two objects are similar (eg, banana and orange), (2) verbal fluency, whereby the participant must 
Table 1 Schedule of assessments

\begin{tabular}{|c|c|c|c|c|c|c|c|c|c|c|c|c|}
\hline & Screening & Bas & ine & & & & & & & & & \\
\hline Visit \# & $1 a$ & $2 a$ & $2 b$ & TC1 & TC2 & TC3 & 3 & 4 & 5 & 6 & 7 & 8 \\
\hline Week & -8 to 0 & -2 & 0 & 2 & 4 & 6 & 8 & 16 & 26 & 39 & 52 & 56 \\
\hline Assess eligibility & $x$ & & & & & & & & & & & \\
\hline Confirmation of eligibility & & & $\mathrm{X}$ & & & & & & & & & \\
\hline Confirmation of Dx of PSP & $x$ & & & & & & & & & & & \\
\hline MRI scan & $x$ & & & & & & & & & & $X$ & \\
\hline tau PET scan & & $x$ & & & & & & & & & $x$ & \\
\hline Lumbar puncture & & $X$ & & & & & & & & & $X$ & \\
\hline Physical examination & $x$ & & $x$ & & & & $\mathrm{X}$ & $\mathrm{X}$ & $\mathrm{X}$ & $X$ & $x$ & $\mathrm{x}$ \\
\hline Neurological examination & $x$ & & $\mathrm{X}$ & & & & & & $\mathrm{X}$ & & $\mathrm{X}$ & \\
\hline Oculomotor testing (optional) & & & $\mathrm{X}$ & & & & & & $X$ & & $X$ & \\
\hline PSP rating scale & & & $\mathrm{X}$ & & & & & & $\mathrm{X}$ & & $\mathrm{X}$ & \\
\hline CGI-C & & & $\mathrm{X}$ & & & & & & $\mathrm{X}$ & & $\mathrm{X}$ & \\
\hline Frontal assessment battery & & & $X$ & & & & & & $\mathrm{X}$ & & $\mathrm{X}$ & \\
\hline Trails $A$ and $B$ & & & $\mathrm{X}$ & & & & & & $\mathrm{X}$ & & $X$ & \\
\hline Digit span (forward and reverse) & & & $X$ & & & & & & $X$ & & $X$ & \\
\hline COWAT and CFT & & & $\mathrm{X}$ & & & & & & $\mathrm{X}$ & & $\mathrm{X}$ & \\
\hline Victoria Stroop & & & $X$ & & & & & & $X$ & & $X$ & \\
\hline Biochemistry & $\mathrm{X}$ & & $x$ & & & & $x$ & & $\mathrm{X}$ & $\mathrm{X}$ & $\mathrm{X}$ & $\mathrm{x}$ \\
\hline Coagulation & $X$ & & & & & & & & & $\mathrm{X}$ & & \\
\hline Blood collected for future exploratory assessments & & $\mathrm{X}$ & & & & & $\mathrm{X}$ & & $\mathrm{X}$ & & $\mathrm{X}$ & \\
\hline Blood collected for pharmacokinetic analysis & & & $\mathrm{X}$ & & & & $\mathrm{X}$ & & & & $\mathrm{X}$ & \\
\hline Urinalysis (dipstick) & $\mathrm{X}$ & & $\mathrm{X}$ & & & & $\mathrm{X}$ & & $\mathrm{X}$ & $\mathrm{X}$ & $\mathrm{X}$ & $\mathrm{X}$ \\
\hline Urine pregnancy test & & & $X$ & & & & $\mathrm{X}$ & $\mathrm{X}$ & $\mathrm{X}$ & $X$ & $\mathrm{X}$ & $\mathrm{X}$ \\
\hline Plasma hCG pregnancy test & $\mathrm{X}$ & & & & & & & & & & & \\
\hline Dispense drug & & & $X$ & & & & & $\mathrm{X}$ & $X$ & $X$ & & \\
\hline Redispense drug & & & & & & & $\mathrm{X}$ & & & & & \\
\hline Study drug administration in clinic & & & $X$ & & & & $\mathrm{X}$ & & & & & \\
\hline Dispense diary card & & & $\mathrm{X}$ & & & & $\mathrm{X}$ & $\mathrm{X}$ & $\mathrm{X}$ & $\mathrm{X}$ & $\mathrm{X}$ & \\
\hline Review of diary card & & & & $\mathrm{X}$ & $X$ & $\mathrm{X}$ & $\mathrm{X}$ & $\mathrm{X}$ & $X$ & $\mathrm{X}$ & $\mathrm{X}$ & $\mathrm{X}$ \\
\hline Review of AEs/SAEs & & & $\mathrm{X}$ & $\mathrm{X}$ & $\mathrm{X}$ & $\mathrm{X}$ & $x$ & $\mathrm{X}$ & $\mathrm{X}$ & $x$ & $\mathrm{X}$ & $\mathrm{x}$ \\
\hline Review of concomitant medications & $\mathrm{X}$ & & $\mathrm{X}$ & $X$ & $X$ & $X$ & $X$ & $X$ & $X$ & $\mathrm{X}$ & $X$ & $\mathrm{X}$ \\
\hline
\end{tabular}

BRIEF-A, Behaviour Rating Inventory of Executive Function A; CFT, Category Fluency Test; CGI-C, clinical global impression of change; COWAT, Controlled Oral Word Association Test; C-SSRS, Colombia Suicide Severity Rating Scale; EQ-5D, European Quality of Life, 5 Dimensions; hCG, human chorionic gonadotropin; PET, positron emission tomography; PSP, progressive supranuclear palsy; SAE, serious adverse event. 
name as many words as they can that begin with a particular letter in $60 \mathrm{~s}$, (3) programming, whereby patients are asked to copy then repeat a series of motor acts, (4) conflicting instructions, whereby the participant is asked to tap the table once when the examiner taps it twice, and tap the table twice when the examiner taps it once. After a practice, the trial consists of a series of 10, (5) go/no-go, whereby the participant is instructed to tap the table once if the examiner does so, but do nothing if the examiner taps twice, (6) prehension behaviour, the examiner places the participants hands palms up on their knees and instructs them to do nothing, the examiner then places their hands on the participant's palms and scores according to the participant's response. The score is computed from summing each of the task scores (range $0-18)$.

\section{Category Fluency Test}

The Category Fluency Test is a test of verbal fluency that measures the participant's ability to spontaneously produce words that belong to a specific category (animals). The participant scored on the number of words they can correctly name in $1 \mathrm{~min}$ that belong to that category.

\section{Controlled Oral Word Association Test}

The Controlled Oral Word Association Test is another test of verbal fluency that also measures executive function. Participants are given a letter of the alphabet and asked to name as many words, within the bounds of test rules (no proper nouns, no repetitions, no identical stem words), that begin with that letter as they can in $1 \mathrm{~min}$. The test is administered three times with three different letters. They are scored on the number of correct responses over the three trials. The whole examination usually takes up to 5 min. $^{33}$

\section{Digit span}

The digit span test measures both attention and working memory. A sequence of digits is read to the participant, which the participant must then repeat back to the examiner. The length of the digit sequences becoming increasing longer over the test. The test is administered both forwards and backwards, with two trials presented at each string length. The score is the sum of correct trials repeated under the two conditions. ${ }^{34}$

\section{Victoria Stroop Test}

Executive function is measured by the Victoria Stroop Test. Three test conditions are used whereby the participant must name the colour of the ink of the stimulus presented. In the first condition they are presented with dots, in the second neutral words and the third incongruent colours. There are 24 items for each condition. ${ }^{35}$

\section{Hayling Sentence Test}

The Hayling Sentence Completion test measures both response initiation and response suppression. The test is entirely verbal, meaning it can be administered to patients who have impairments in reading or visual perception such as those with PSP. The test involves two series of 15 sentences which are missing the final word. For the first part the examiner reads each sentence aloud which the participant must complete as quickly as they can, thus generating a measure of the speed of response initiation. In the second half of the test, the participant must again complete the sentence read to them, but this time with a non-sensical ending, which measures the ability to suppress responses as well as thinking time. The test administration takes approximately $5 \mathrm{~min}$. The test produces three measures of executive function that can be used alone or in combination. ${ }^{36}$

\section{NIH toolbox}

The NIH toolbox cognitive battery consists of a number of cognitive tests that can be used alone or in combination to assess global cognitive function. It has been designed for longitudinal measurement of participants' function and is thus validated for repeated administration. The cognitive battery takes approximately $30 \mathrm{~min}$ to complete. $^{37}$

\section{European Quality of Life, 5 Dimensions, 5 Levels}

The European Quality of Life, 5 Dimensions, 5 Levels is a quality of life scale that comprises five dimensions: mobility, self-care, usual activities, pain/discomfort and anxiety/depression. Each dimension has five levels: no problems, slight problems, moderate problems, severe problems and extreme problems, expressed as a single digit (range 1-5). ${ }^{38}$ The participant's health state is the combination of these digits in to a 5-digit number. The participant is also asked to mark their health on a visual analogue scale (range 0-100).

\section{Behaviour Rating Inventory of Executive Function A}

The Behaviour Rating Inventory of Executive Function A is a participant-administered and informant-administered scale which assesses executive function. ${ }^{39}$ In instances where the participant is unable or has limited awareness of their own difficulties, the informant-only report may be used.

The assessment covers nine areas of executive functioning: inhibition, self-monitoring, planning and organisation, attention shifting, initiative task monitoring, emotional control, working memory and organisation of materials. These areas form two broad indices: behavioural regulation and metacognition, as well as an overall global executive composite.

\section{OM testing}

In participants who consent to the OM substudy, testing will be carried out at baseline, week 26 and week 52. Horizontal and vertical displacement of both eyes will be recorded using the Saccadometer Research Advanced (Ober Consulting) or the Eyelink 1000 plus dark pupil video-oculography system at a sample frequency of $1000 \mathrm{~Hz}$. These systems incorporate built-in visual targets using miniature laser projectors mounted on a sensor forehead plate, and records average movement of both eyes in response to a number of preprogrammed trials. Participants will be seated $50 \mathrm{~cm}$ from 
a blank wall. Experimental trials will include: Horizontal saccades (test of basic OM function)-participants will generate saccades to targets that step from centre fixation to either $5^{\circ}$ or $10^{\circ}$ left or right of centre; vertical saccades (test of basic $\mathrm{OM}$ function)-participants will generate saccades to targets that step from centre fixation to either $5^{\circ}$ or $10^{\circ}$ directly above or below centre; antisaccades (test of inhibitory/executive control)—participants will generate saccades in the mirror-opposite location of targets that step from centre fixation to either $5^{\circ}$ or $10^{\circ}$ left or right of centre; memory-guided saccades (test of working memory) - participants will generate saccades to the remembered location of a previously presented target that appeared at either $5^{\circ}$ or $10^{\circ}$ left or right of centre; fixation (test of basic OM function) participants will maintain fixation on a stationary target that appears at centre or $5^{\circ}$ or $10^{\circ}$ left or right of centre.

For each task the measures of interest will include: saccade latency, saccade velocity, saccade accuracy and task error. Test results will be stored in the device's memory, downloaded and transferred to the central laboratory to be collated and analysed.

\section{Safety assessments}

Each clinic visit will include the following safety assessments: physical examination, 12-lead ECG and haematology, chemistry and urinalysis (all visits except week 16). Neurological examinations will also be completed at screening, baseline, week 26 and 52. The results of these investigations will be reviewed and clinically significant abnormalities will be documented as adverse events. At each clinic visit the participant and their study partner will be asked about any adverse events or concomitant medications, and any adverse events recorded on the diary cards confirmed and recorded. ${ }^{26}$

\section{Blood biomarkers}

Pharmacokinetic, biomarker and exploratory blood samples will be collected as detailed in table 1 . Blood samples (6mL/sample) for pharmacokinetics will be taken predose ( 1 hour), then $0.5,1,2$ and 4 hours after dosing at the baseline, week 8 , and week 52 visits. Plasma will be stored at $-80^{\circ} \mathrm{C}$ until measurement of sodium selenate levels for establishing the pharmacokinetic profile.

Plasma and serum samples will be taken at baseline, week 8, week 26 and week 52 for exploratory analyses. Additional samples for DNA and RNA will be taken only at baseline. Biomarkers of neurodegeneration including total-tau, phospho-tau, neurofilament light, as well as testing for genes associated with PSP (MAPT, LRRK2) will be included in the exploratory analyses. Additional hypothesis driven testing may be performed. ${ }^{26}$

\section{CSF biomarkers}

Sampling of CSF will be performed at baseline (pretreatment) and week 52. Approximately $20 \mathrm{~mL}$ of CSF will be collected using atraumatic needles (20G) and polypropylene tubes $(10 \mathrm{~mL})$ cooled on ice. Samples will remain on ice until they are aliquoted in to $500 \mu \mathrm{L}$ polypropylene aliquots. Samples will be stored at $-80^{\circ} \mathrm{C}$ until analysis. Planned analyses will measure the proteins total-tau, phospho-tau and NfL. Additional testing of CSF will be performed as new research questions emerge.

\section{Power and sample size}

The study is powered to detect a difference in the primary outcome and will therefore be declared positive or negative on the primary outcome measure. The sample size has been determined on the primary outcome variable (MRI composite volume). The annual rate of change of this composite in PSP-RS is $-12.9 \% \quad(\mathrm{SD}=7.1) .{ }^{28}$ The mean atrophy rate in controls is $3.76 \% .^{40}$ A sample size of 46 patients (randomised 1:1 into two groups) would be sufficient to detect a medium effect size (Cohen's $\mathrm{d}=0.50$, alpha $=5 \%$, power $=80 \%$ ). This equates to detecting a $7 \%$ rate of atrophy, which is a $46 \%$ reduction in atrophy rate compared with the natural history, which will represent a clinically meaningful treatment effect.

Trials in PSP have high withdrawal rates. Recruitment of 70 participants will allow for up to $30 \%$ attrition while ensuring the study remains adequately powered. Previous studies have demonstrated the safety and tolerability of sodium selenate, for this reason there will be no interim safety, efficacy or futility analyses.

\section{Outcomes and statistical overview Primary endpoint}

The primary endpoint measure is the change in MRI volume composite (frontal lobe+midbrain-3rd ventricle) from baseline to week 52 between treatment and placebo groups. The primary analysis will include all participants with a post-baseline MRI. Statistical analysis will use a GLM, with the MRI composite at week 52 as the dependent variable, treatment group as an independent variable, and baseline MRI composite as the covariate.

\section{Secondary endpoints}

Descriptive statistics (mean, median, minimum, maximum, SD) by visit will report all continuous secondary efficacy endpoints. Data transformations (such as change and percentage change) will be summarised similarly.

The change from baseline to week 52 in PSPRS, CGI-C and midbrain MD (measured on DWI) will be analysed using a GLM which includes the respective baseline measure as a covariate in the model. The model will estimate the adjusted mean change (with $95 \%$ confidence limits) as a marker of treatment.

\section{Safety and tolerability}

Safety and tolerability measures will be presented as frequency tables of categorical outcomes by visit (number of participants and percentage). Tables will demonstrate both the number of participants affected $(\mathrm{N})$ and the number of incidences $(n)$.

Determination of safety and tolerability will be by the frequency of serious adverse events ((SAEs), Common Terminology Criteria for Adverse Events score $\geq 3$ ), 
frequency of down-titration events and frequency of study discontinuation. ${ }^{26}$

\section{Monitoring and data quality}

In accordance with the International Conference on Harmonisation Good Clinical Practice guidelines, source data verification will be completed by the project manager at regular intervals throughout the study to ensure the eCRF remains up to date, accurate and reliable. The rate of subject recruitment will determine monitoring visit frequency. ${ }^{26}$

Safety oversight will be provided by an independent medical monitor, who will oversee the study conduct and regularly (every 3 months) review all safety-related events.

The DSMB will be made up of an independent clinician, an independent biostatistician and the medical monitor. The DSMB meetings will begin within 2 weeks of the week 8 visit for the third randomised patient or within 1 week of the second SAE occurring, whichever is first. Subsequently meetings will be at 6 monthly intervals. The medical monitor or site principal investigator may request additional DSMB meetings should there be urgent safety concerns. The medical monitor will make recommendations to the principal investigator based on the safety and tolerability issues after each DSMB review. ${ }^{26}$

\section{Patient and public involvement}

Study conception and design did not involve patients. As is required by Australian ethics committees and stated in the study consent forms, a plain English summary of the study will be provided to all study participants (and their person responsible/study partner) at the conclusion of the study. Wider dissemination of the results of the study to the community will be done via the media, patient support groups such as Parkinson's Australia and PSP Australia, and open events at our hospitals and research institutes.

\section{ETHICS AND DISSEMINATION}

Ethics approval for the study has been granted by the Alfred Hospital Ethics Committee (HREC, 594/20). All participants or their legally authorised representative and their study partner will provide written informed consent prior to commencement of any study assessments. An example form is available as online supplemental file 1 . The study results will be disseminated through presentations at national and international conferences and published in peer-reviewed journals. Any protocol amendments will be approved by the HREC prior to implementation and subsequently updated on ANZCTR.

\footnotetext{
Author affiliations

${ }^{1}$ Department of Neurosciences, Monash University, Melbourne, Victoria, Australia

${ }^{2}$ Department of Neurology, Alfred Health, Melbourne, Victoria, Australia

${ }^{3}$ Department of Neurology, Royal Melbourne Hospital, The University of Melbourne, Parkville, Victoria, Australia

${ }^{4}$ Department of Medicine, University of Melbourne, Melbourne, Victoria, Australia ${ }^{5}$ Melbourne School of Psychological Sciences, University of Melbourne, Melbourne, Victoria, Australia
}

${ }^{6}$ ForeFront Parkinson's Disease Research Clinic, Brain and Mind Centre, University of Sydney, Sydney, New South Wales, Australia

${ }^{7}$ Department of Neurology, St Vincent's Hospital Sydney, Darlinghurst, New South Wales, Australia

${ }^{8}$ Centre for Clinical Research, University of Queensland, Brisbane, Queensland, Australia

${ }^{9}$ Department of Neurology, Royal Brisbane \& Women's Hospital, Brisbane, Queensland, Australia

${ }^{10}$ Department of Neurology, Royal Adelaide Hospital, Adelaide, South Australia, Australia

${ }^{11}$ The Florey Institute of Neuroscience and Mental Health, Melbourne, Victoria, Australia

${ }^{12}$ Eastern Cognitive Disorders Clinic, Box Hill Hospital, Melbourne, Victoria, Australia

${ }^{13}$ Department of Radiology, Alfred Hospital, Melbourne, Victoria, Australia

${ }^{14}$ Department of Surgery, Royal Melbourne Hospital, The University of Melbourne, Parkville, Victoria, Australia

${ }^{15}$ Department of Neuropsychiatry, Royal Melbourne Hospital, Parkville, Victoria, Australia

${ }^{16}$ Melbourne Neuropsychiatry Centre, University of Melbourne, Parkville, Victoria, Australia

Contributors LV drafted the manuscript. LV, KLB, CMalpas, CMarotta, IHH, SK, JF, MC, SJGL, ST, AHE, JDOS, TK, LC, DD, ML, CMH, DV and TJOB contributed to the study design. All authors edited the manuscript and approved the final version.

Funding This work is supported by the Australian National Health and Medical Research Council (NHMRC) Medical Research Future Fund grant number GNT1200254. TJOB is supported by an NHMRC Investigator Grant (APP1176426). SJGL is supported by NHMRC Leadership Fellowship (\#1195830).

Competing interests All authors report a grant from the NHMRC to support this study. The authors report the following disclosures outside of this study: LV reports personal fees from Biogen Australia, and research funding from Biogen, Eisai and LMI. AHE reports honoraria for presentations from Merck, Allergan, Ipsen, Teva, UCB, Abbott, AbbVie, STADA, participation in scientific advisory board meetings with Allergan, AbbVie, Ipsen and STADA and shares in GKC and CSL. DD reports consultancy fees from Biogen, Novartis. KLB, CMalpas, CMarotta, IHH, SK, JF, MC, SJGL, ST, JDOS, TK, LC, ML, CMH and DV report no disclosures.

Patient consent for publication Not applicable.

Provenance and peer review Not commissioned; externally peer reviewed.

Supplemental material This content has been supplied by the author(s). It has not been vetted by BMJ Publishing Group Limited (BMJ) and may not have been peer-reviewed. Any opinions or recommendations discussed are solely those of the author(s) and are not endorsed by BMJ. BMJ disclaims all liability and responsibility arising from any reliance placed on the content. Where the content includes any translated material, BMJ does not warrant the accuracy and reliability of the translations (including but not limited to local regulations, clinical guidelines, terminology, drug names and drug dosages), and is not responsible for any error and/or omissions arising from translation and adaptation or otherwise.

Open access This is an open access article distributed in accordance with the Creative Commons Attribution Non Commercial (CC BY-NC 4.0) license, which permits others to distribute, remix, adapt, build upon this work non-commercially, and license their derivative works on different terms, provided the original work is properly cited, appropriate credit is given, any changes made indicated, and the use is non-commercial. See: http://creativecommons.org/licenses/by-nc/4.0/.

ORCID iD

Lucy Vivash http://orcid.org/0000-0002-1182-0907

\section{REFERENCES}

1 Schrag A, Ben-Shlomo Y, Quinn NP. Prevalence of progressive supranuclear palsy and multiple system atrophy: a cross-sectional study. Lancet 1999;354:1771-5.

2 O'Sullivan SS, Massey LA, Williams DR, et al. Clinical outcomes of progressive supranuclear palsy and multiple system atrophy. Brain 2008;131:1362-72.

3 Höglinger GU, Litvan I, Mendonca N, et al. Safety and efficacy of tilavonemab in progressive supranuclear palsy: a phase 2 , randomised, placebo-controlled trial. Lancet Neurol 2021;20:182-92. 
4 Bensimon G, Ludolph A, Agid Y, et al. Riluzole treatment, survival and diagnostic criteria in Parkinson plus disorders: the NNIPPS study. Brain 2009;132:156-71

5 Williams DR, Lees AJ. Progressive supranuclear palsy: clinicopathological concepts and diagnostic challenges. Lancet Neurol 2009;8:270-9.

6 Goedert M, Jakes R, Qi Z, et al. Protein phosphatase 2A is the major enzyme in brain that dephosphorylates tau protein phosphorylated by proline-directed protein kinases or cyclic AMP-dependent protein kinase. J Neurochem 1995;65:2804-7.

7 Arnold SE, Hyman BT, Flory J, et al. The topographical and neuroanatomical distribution of neurofibrillary tangles and neuritic plaques in the cerebral cortex of patients with Alzheimer's disease. Cereb Cortex 1991;1:103-16.

8 Hyman BT, Van Hoesen GW, Damasio AR, et al. Alzheimer's disease: cell-specific pathology isolates the hippocampal formation. Science 1984;225:1168-70.

9 Gong CX, Lidsky T, Wegiel J, et al. Phosphorylation of microtubuleassociated protein tau is regulated by protein phosphatase $2 \mathrm{~A}$ in mammalian brain. Implications for neurofibrillary degeneration in Alzheimer's disease. J Biol Chem 2000;275:5535-44.

10 Corcoran NM, Najdovska M, Costello AJ. Inorganic selenium retards progression of experimental hormone refractory prostate cancer. $J$ Urol 2004;171:907-10.

11 Brady RD, Grills BL, Romano T, et al. Sodium selenate treatment mitigates reduction of bone volume following traumatic brain injury in rats. J Musculoskelet Neuronal Interact 2016;16:369.

12 Corcoran NM, Hovens CM, Michael M, et al. Open-Label, phase I dose-escalation study of sodium selenate, a novel activator of PP2A, in patients with castration-resistant prostate cancer. $\mathrm{Br} \mathrm{J}$ Cancer 2010;103:462-8.

13 Corcoran NM, Martin D, Hutter-Paier B, et al. Sodium selenate specifically activates PP2A phosphatase, dephosphorylates tau and reverses memory deficits in an Alzheimer's disease model. J Clin Neurosci 2010;17:1025-33.

14 lqbal K, Alonso AdelC, Chen S, et al. Tau pathology in Alzheimer disease and other tauopathies. Biochim Biophys Acta 2005;1739:198-210.

15 Jones NC, Nguyen T, Corcoran NM, et al. Targeting hyperphosphorylated tau with sodium selenate suppresses seizures in rodent models. Neurobiol Dis 2012;45:897-901.

16 Liu S-J, Zheng P, Wright DK, et al. Sodium selenate retards epileptogenesis in acquired epilepsy models reversing changes in protein phosphatase $2 \mathrm{~A}$ and hyperphosphorylated tau. Brain 2016;139:1919-38.

17 Malpas CB, Vivash L, Genc S, et al. A phase lla randomized contro trial of VEL015 (Sodium Selenate) in mild-moderate Alzheimer's disease. J Alzheimers Dis 2016;54:223-32.

18 Shultz SR, Wright DK, Zheng P, et al. Sodium selenate reduces hyperphosphorylated tau and improves outcomes after traumatic brain injury. Brain 2015;138:1297-313.

19 Tan XL, Wright DK, Liu S, et al. Sodium selenate, a protein phosphatase $2 \mathrm{~A}$ activator, mitigates hyperphosphorylated tau and improves repeated mild traumatic brain injury outcomes. Neuropharmacology 2016;108:382-93.
20 Whitehouse PJ, Price DL, Struble RG, et al. Alzheimer's disease and senile dementia: loss of neurons in the basal forebrain. Science 1982;215:1237-9.

21 Zheng P, Shultz SR, Hovens CM, et al. Hyperphosphorylated tau is implicated in acquired epilepsy and neuropsychiatric comorbidities. Mol Neurobiol 2014;49:1532-9

22 van Eersel J, Ke YD, Liu X, et al. Sodium selenate mitigates tau pathology, neurodegeneration, and functional deficits in Alzheimer's disease models. Proc Natl Acad Sci U S A 2010;107:13888-93.

23 Cardoso BR, Roberts BR, Malpas CB, et al. Supranutritional sodium selenate supplementation delivers selenium to the central nervous system: results from a randomized controlled pilot trial in Alzheimer's disease. Neurotherapeutics 2019;16:192-202.

24 Vivash L, Malpas C, Hovens A, et al. Sodium selenate as a disease modifying treatment for mild-moderate Alzheimer's Disease: an open label extension study. BMJ Neurology Open; In press.

25 Chare L, Hodges JR, Leyton CE, et al. New criteria for frontotemporal dementia syndromes: clinical and pathological diagnostic implications. J Neurol Neurosurg Psychiatry 2014;85:865-70.

26 Vivash L, Malpas CB, Churilov L, et al. A study protocol for a phase II randomised, double-blind, placebo-controlled trial of sodium selenate as a disease-modifying treatment for behavioural variant frontotemporal dementia. BMJ Open 2020;10:e040100.

27 Höglinger GU, Respondek G, Stamelou M, et al. Clinical diagnosis of progressive supranuclear palsy: the movement disorder Society criteria. Mov Disord 2017;32:853-64.

28 Höglinger GU, Schöpe J, Stamelou M, et al. Longitudinal magnetic resonance imaging in progressive supranuclear palsy: a new combined score for clinical trials. Mov Disord 2017;32:842-52.

29 Golbe LI, Ohman-Strickland PA. A clinical rating scale for progressive supranuclear palsy. Brain 2007;130:1552-65.

30 Guy W. Clinical Global Impressions. In: ECDEU assessment manual for psychopharmacology. Rockville MD: US Department of Health, Education and Welfare, 1976: 218-22.

31 Tombaugh TN. Trail making test $A$ and $B$ : normative data stratified by age and education. Arch Clin Neuropsychol 2004;19:203-14.

32 Dubois B, Slachevsky A, Litvan I, et al. The Fab: a frontal assessment battery at bedside. Neurology 2000;55:1621-6.

33 Benton AL, Hamsher KdeS. Multilingual aphasia examination: Mnaul of instruction. lowa City: University of lowa, 1976.

34 Wechsler D. Wechsler adult intelligence scale. Vol. 22. Fourth Edition WAIS-IV. San Antonio, TX: NCS Pearson, 2008.

35 Spreen O, Strauss E. A compendium of neuropsychological tests: Administration, norms and commentary. 2 ed. New York: Oxford University Press, 1998.

36 Burgess PaS. T. Hayling and Brixton tests. Pearson, 1997.

37 Weintraub S, Dikmen SS, Heaton RK, et al. Cognition assessment using the NIH Toolbox. Neurology 2013;80:S54-64.

38 Herdman M, Gudex C, Lloyd A, et al. Development and preliminary testing of the new five-level version of EQ-5D (EQ-5D-5L). Qual Life Res 2011;20:1727-36.

39 Roth RMet al. Development of the behavior rating inventory of executive Function-Adult version. Arch Clin Neuropsychol 2005;20:906.

40 Takao H, Hayashi N, Ohtomo K. A longitudinal study of brain volume changes in normal aging. Eur J Radiol 2012;81:2801-4. 